\title{
The cosmic web in CosmoGrid void regions
}

\section{Steven Rieder ${ }^{1,2}$, Rien van de Weygaert ${ }^{1}$, Marius Cautun ${ }^{3,1}$, Burcu Beygu ${ }^{1}$ and Simon Portegies Zwart ${ }^{2}$}

${ }^{1}$ Kapteyn Instituut, Rijksuniversiteit Groningen, P.O. Box 800, 9700 AV Groningen, The Netherlands, email: steven@stevenrieder.nl

2 Sterrewacht Leiden, Leiden University, P.O. Box 9513, 2300 RA Leiden, The Netherlands,

${ }^{3}$ Department of Physics, Institute for Computational Cosmology, University of Durham, South Road, Durham DH1 3LE, UK

\begin{abstract}
We study the formation and evolution of the cosmic web, using the high-resolution CosmoGrid $\Lambda \mathrm{CDM}$ simulation. In particular, we investigate the evolution of the large-scale structure around void halo groups, and compare this to observations of the VGS-31 galaxy group, which consists of three interacting galaxies inside a large void.

The structure around such haloes shows a great deal of tenuous structure, with most of such systems being embedded in intra-void filaments and walls. We use the Nexus+ algorithm to detect walls and filaments in CosmoGrid, and find them to be present and detectable at every scale. The void regions embed tenuous walls, which in turn embed tenuous filaments. We hypothesize that the void galaxy group of VGS-31 formed in such an environment.
\end{abstract}

Keywords. large-scale structure of universe, dark matter

\section{Introduction}

The large-scale structure of the Universe is volume-dominated by voids: enormous regions of space that contain very few galaxies (see Zeldovich et al. 1982, and van de Weygaert and Platen 2011 for a review). They are surrounded by walls, filaments and clusters, together forming the Cosmic Web (Bond et al. 1996). Kreckel et al. (2011, 2012) conducted a survey of 60 void galaxies, selected from the SDSS. One of these, VGS-31, was later revealed to be an interacting galaxy system, consisting of three galaxies that appeared to be aligned along an HI filament (Beygu et al. 2013). It is a manifestation of void substructure as a result of hierarchical evolution (van de Weygaert and van Kampen 1993; Sheth and van de Weygaert 2004; Aragon-Calvo et al. 2010; Aragon-Calvo and Szalay 2013).

\section{Simulation and methods}

In order to investigate the formation of a system like VGS-31, we study the environment of dark matter haloes in cosmic voids within the high-resolution CosmoGrid $\Lambda$ CDM suite of simulations (Portegies Zwart et al. 2010; Ishiyama et al. 2013). CosmoGrid was performed using the GreeM/SUSHI code (Ishiyama et al. 2009; Groen et al. 2011). The most detailed realisation contains $2048^{3}$ particles in a $(30 \mathrm{Mpc})^{3}$ volume, resulting in a mass of $1.28 \times 10^{5} \mathrm{M}_{\odot}$ for each particle. With a softening length $\epsilon$ of 175 parsec at $z=0$ and over 500 snapshots, it has a very high mass-, spatial- and time-resolution.

Due to its high resolution, CosmoGrid is especially suitable for case studies of smaller dark matter haloes (up to Milky Way and Local Group-size). However, its limited volume makes it less suited for studies requiring statistical representation. 

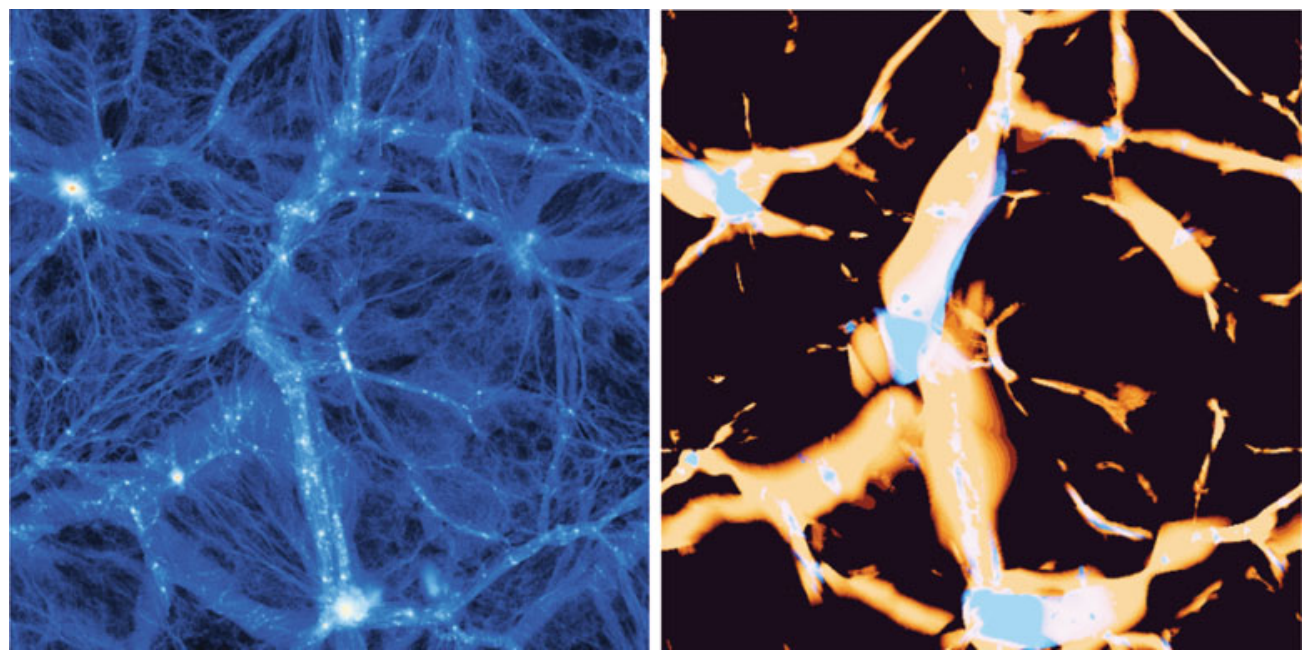

Figure 1. $600 \mathrm{kpc}$ thick slice of the $1024^{3}$-particle CosmoGrid realization at $\mathrm{z}=0$. Left: particles. Right: Nexus+ analysis of the same region. Blue regions represent filaments and clusters, while orange represents the walls.

For the analysis of the CosmoGrid environment, we use the Nexus+ (Cautun et al. 2013, 2014) and DTFE (Schaap and van de Weygaert 2000; van de Weygaert and Schaap 2009; Cautun and van de Weygaert 2011) tools and the AMUSE (Portegies Zwart et al. 2013; Pelupessy et al. 2013) framework. DTFE uses the Voronoi and Delaunay tessellations of the particle distribution to construct a volume-weighted linear piecewise density field. The inverse volume of the contiguous Voronoi cells provide a local density estimate, and the Delaunay tessellation are used as an interpolation grid. The resulting field reconstruction retains the anisotropic character and multiscale structure of the particle distribution. We use DTFE to determine densities in the volume (smoothed on a scale of $1 h^{-1} \mathrm{Mpc}$ ), where we identify regions with a density contrast $\delta<0$ as void regions.

Additionally, we use Nexus+ to determine whether a region is part of a wall, a filament or a cluster. Nexus+ is a technique for the multiscale morphological characterisation of the spatial mass distribution. It is an extension and improvement of the Multiscale Morphology Filter (Aragón-Calvo et al. 2007), in which the locally dominant morphological signal is extracted from the $4 \mathrm{~d}$ scale-space representation of the mass distribution. It dissects the mass distribution into voids, walls, filaments and cluster nodes on the basis of the local signature of the Hessian of the logarithm of the density field. Using this method, we analyse the full volume of CosmoGrid (see figure 1). Due to the multi-scale nature of the cosmic environment, a void region found with a $1 h^{-1} \mathrm{Mpc}$ smoothing scale can still contain walls, filaments and clusters on a smaller scale.

\section{VGS-31-like systems in CosmoGrid}

\subsection{Candidate systems}

We use CosmoGrid data to investigate possible formation scenarios for the VGS-31 system, and to find out if the filamentary structure seen in the alignment of these galaxies is to be expected or coincidental. To this end, we first search for systems of similar properties to VGS-31 in CosmoGrid. Using the mass, environmental density contrast and size of the system as selection criteria, we find eight candidate systems in CosmoGrid that we name "CosmoGrid Void Systems" (CGV, see table 1 and Rieder et al. 2013, for details). 


\begin{tabular}{llllcc}
\hline Name & $M_{\text {vir }}\left(h^{-1} \mathrm{M}_{\odot}\right)$ & Last MM $(\mathrm{Gyr})$ & $\delta$ & Wall & Filament \\
\hline CGV-A & $3.15 \times 10^{10}$ & - & -0.68 & $\mathrm{X}$ & - \\
CGV-B & $3.95 \times 10^{10}$ & 5.24 & -0.51 & $\mathrm{X}$ & $\mathrm{X}$ \\
CGV-C & $2.99 \times 10^{10}$ & 1.19 & -0.51 & $\mathrm{X}$ & - \\
CGV-D & $4.60 \times 10^{10}$ & 10.9 & -0.63 & $\mathrm{X}$ & $\mathrm{X}$ \\
CGV-E & $1.99 \times 10^{10}$ & 2.44 & -0.57 & $\mathrm{X}$ & $\mathrm{X}$ \\
CGV-F & $2.27 \times 10^{10}$ & - & -0.62 & $\mathrm{X}$ & $\mathrm{X}$ \\
CGV-G & $2.14 \times 10^{10}$ & 5.80 & -0.61 & $\mathrm{X}$ & $\mathrm{X}$ \\
CGV-H & $4.63 \times 10^{10}$ & 8.45 & -0.50 & $\mathrm{X}$ & - \\
\hline
\end{tabular}

Table 1. VGS-31-like systems in CosmoGrid. 1: Virial mass of the main halo, 2: Time of the last major merger, 3: Density contrast, 4: System is embedded in a wall, 5: System is embedded in a filament.
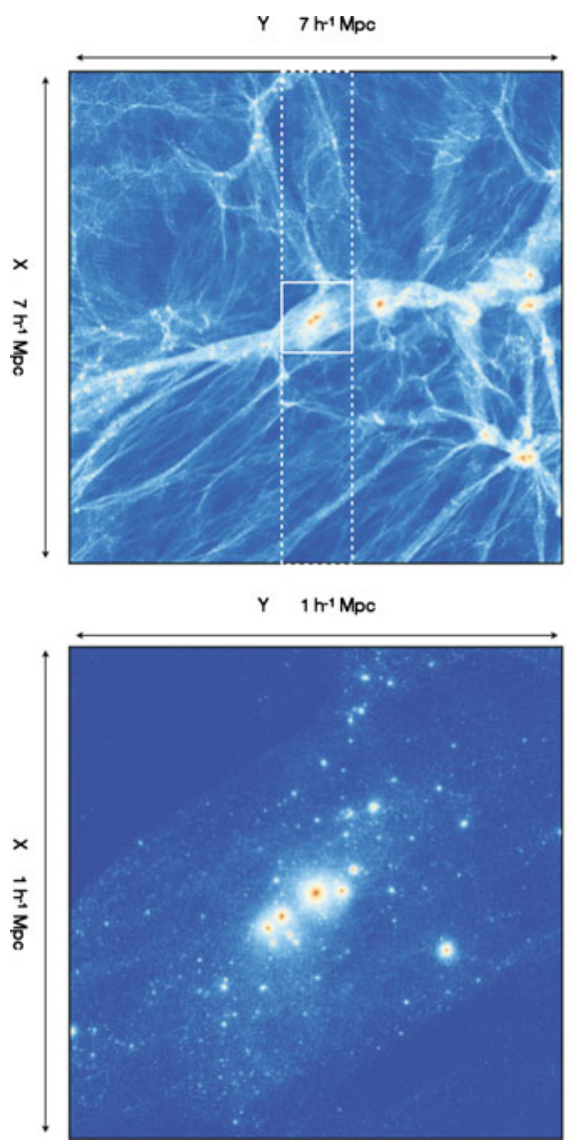

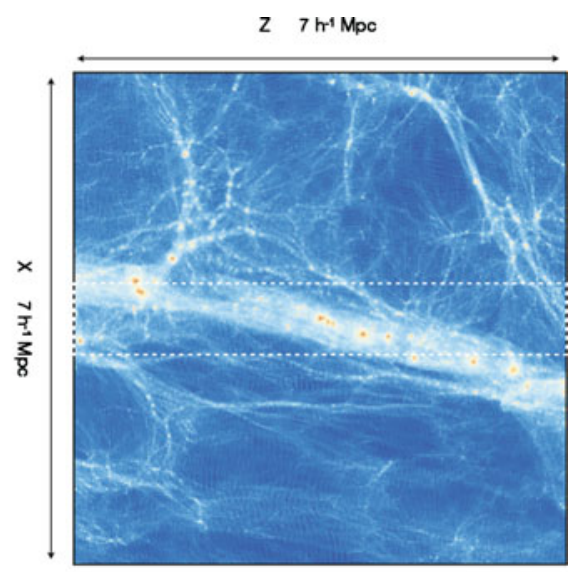

Z $\quad 7 \mathrm{~h}^{-1} \mathrm{Mpc}$

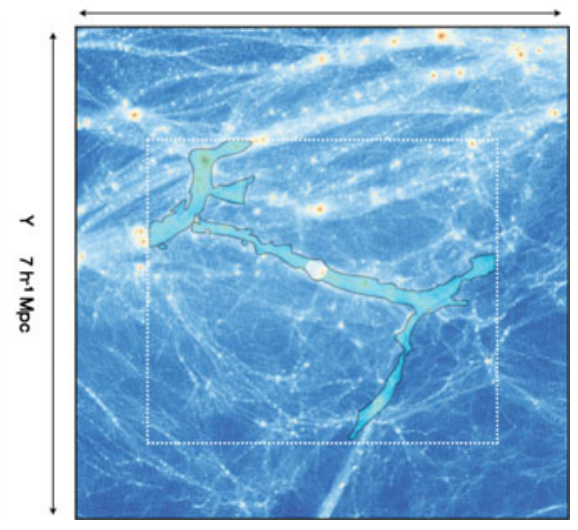

Figure 2. Environment of CGV-G, as seen from different angles, in $1 h^{-1} \mathrm{Mpc}$ thick slices. Lower-right panel includes a Nexus+ overlay.

While at $z=0$ these systems appear very similar, their formation histories are quite diverse: some systems continue to experience mergers up to $z=0$, while others remain virtually unchanged since $z=6$. Here, we discuss the environment of these haloes.

\subsection{Environment of the systems}

In figure 2, we show the environment of CGV-G as a representative for the void halo systems, at a $7 h^{-1} \mathrm{Mpc}$ scale. While the whole system is in a void, its environment is 


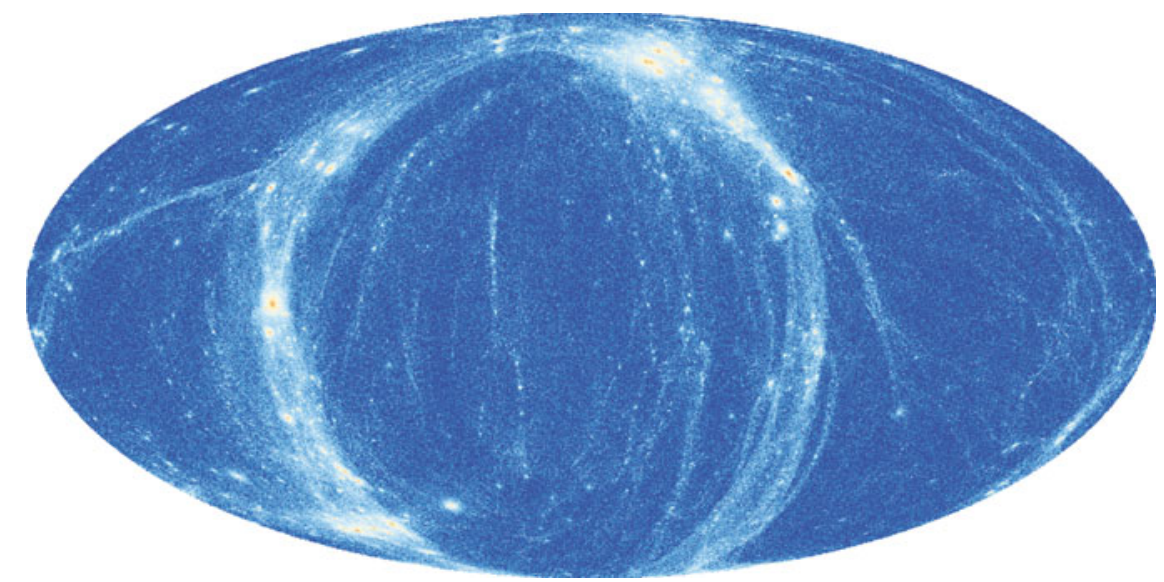

Figure 3. Mollweide projection of the environment around CGV-G at $z=0$, up to a distance of $2 h^{-1} \mathrm{Mpc}$ from the main halo's centre. The wall manifests itself as a circle, while the filament is visible within the wall as two bright regions on opposite sides (top-right and lower-left).

highly structured. The tenuous wall, which in turn contains a thin filament, is clearly visible in this figure.

While the majority of the CGV systems forms along a tenuous filament, all of the systems are embedded in thin but prominent walls (see table 1). These walls have a typical thickness of around $0.4 h^{-1} \mathrm{Mpc}$. They show a strong coherence and retain the character of a highly flattened structure out to a distance of at least $3 h^{-1} \mathrm{Mpc}$ from the CGV haloes. The filaments in which five of the systems reside are rather short, not longer than $4 h^{-1} \mathrm{Mpc}$, with a diameter of around $0.4 h^{-1} \mathrm{Mpc}$. Compared to the high-density filaments of the larger-scale cosmic web, the environment of void haloes is very feeble. At earlier times, this structure inside the void is much more prominent, becoming more tenuous over time until it can hardly be detected at $z=0$. However, it remains visible in the alignment of the haloes. The formation histories of these haloes are quite diverse, with the main haloes forming between $z=6$ and $z=0$.

In figure 3, we show a Mollweide projection of all the dark matter around CGV-G up to a distance of $2 h^{-1} \mathrm{Mpc}$. This projection clearly shows the wall in which the system is embedded, while the filament appears as two bright spots on opposite sides. We see very similar structure around the other CGV-systems.

A similar study within a larger volume will provide a more definitive answer on the abundance of VGS-31-like systems. Furthermore, a continuation study with a hydrodynamical simulation will provide insight into the gaseous surroundings of such systems.

\section{Acknowledgements}

The authors are grateful to Tomoaki Ishiyama and Bernard Jones, for many insightful discussions. This work was supported by NWO grants IsFast [\#643.000.803], VICI [\#639.073.803], LGM [\#612.071.503] and AMUSE [\#614.061.608]), NCF (grants [\#SH095-08] and [\#SH-187-10]), NOVA and the LKBF in the Netherlands. SR and RvdW acknowledge support by the John Templeton Foundation, grant [\#FP05136-O].

\section{References}

M. A. Aragon-Calvo \& A. S. Szalay. The hierarchical structure and dynamics of voids. MNRAS, 428:3409-3424, February 2013. . 
M. A. Aragón-Calvo, B. J. T. Jones, R. van de Weygaert, \& J. M. van der Hulst. The multiscale morphology filter: identifying and extracting spatial patterns in the galaxy distribution. $A \& A, 474: 315-338$, October 2007. .

M. A. Aragon-Calvo, R. van de Weygaert, P. A. Araya-Melo, E. Platen, \& A. S. Szalay. Unfolding the hierarchy of voids. MNRAS, 404:L89-L93, May 2010. .

B. Beygu, K. Kreckel, R. van de Weygaert, J. M. van der Hulst, \& J. H. van Gorkom. An Interacting Galaxy System along a Filament in a Void. AJ, 145:120, May 2013. .

J. R. Bond, L. Kofman, \& D. Pogosyan. How filaments of galaxies are woven into the cosmic web. Nature, 380:603-606, April 1996. .

M. Cautun, R. van de Weygaert, \& B. J. T. Jones. NEXUS: tracing the cosmic web connection. MNRAS, 429:1286-1308, February 2013. .

M. Cautun, R. van de Weygaert, B. J. T. Jones, \& C. S. Frenk. Evolution of the cosmic web. MNRAS, 441:2923-2973, July 2014. .

M. C. Cautun \& R. van de Weygaert. The DTFE public software - The Delaunay Tessellation Field Estimator code. arXiv:1105.0370, May 2011.

D. Groen, S. Portegies Zwart, T. Ishiyama, \& J. Makino. High-performance gravitational Nbody simulations on a planet-wide-distributed supercomputer. Computational Science and Discovery, 4(1):015001-+, January 2011. .

T. Ishiyama, T. Fukushige, \& J. Makino. GreeM: Massively Parallel TreePM Code for Large Cosmological N -body Simulations. PASJ, 61:1319-, December 2009.

T. Ishiyama, S. Rieder, J. Makino, S. Portegies Zwart, D. Groen, K. Nitadori, C. de Laat, S. McMillan, K. Hiraki, \& S. Harfst. The Cosmogrid Simulation: Statistical Properties of Small Dark Matter Halos. ApJ, 767:146, April 2013. .

K. Kreckel, E. Platen, M. A. Aragón-Calvo, J. H. van Gorkom, R. van de Weygaert, J. M. van der Hulst, K. Kovač, C.-W. Yip, \& P. J. E. Peebles. Only the Lonely: H I Imaging of Void Galaxies. AJ, 141:4, January 2011. .

K. Kreckel, E. Platen, M. A. Aragón-Calvo, J. H. van Gorkom, R. van de Weygaert, J. M. van der Hulst, \& B. Beygu. The Void Galaxy Survey: Optical Properties and H I Morphology and Kinematics. AJ, 144:16, July 2012. .

F. I. Pelupessy, A. van Elteren, N. de Vries, S. L. W. McMillan, N. Drost, \& S. F. Portegies Zwart. The Astrophysical Multipurpose Software Environment. A\&GA, 557:A84, September 2013. .

S. Portegies Zwart, T. Ishiyama, D. Groen, K. Nitadori, J. Makino, C. de Laat, S. McMillan, K. Hiraki, S. Harfst, \& P. Grosso. Simulating the universe on an intercontinental grid of supercomputers. IEEE Computer, v.43, No.8, p.63-70, 43:63-70, October 2010. .

S. Portegies Zwart, S. L. W. McMillan, E. van Elteren, I. Pelupessy, \& N. de Vries. Multi-physics simulations using a hierarchical interchangeable software interface. Computer Physics Communications, 183:456-468, March 2013. .

S. Rieder, R. van de Weygaert, M. Cautun, B. Beygu, \& S. Portegies Zwart. Assembly of filamentary void galaxy configurations. MNRAS, 435:222-241, October 2013. .

W. E. Schaap \& R. van de Weygaert. Continuous fields and discrete samples: reconstruction through Delaunay tessellations. A\&A, 363:L29-L32, November 2000.

R. K. Sheth \& R. van de Weygaert. A hierarchy of voids: much ado about nothing. MNRAS, 350:517-538, May 2004.

R. van de Weygaert \& E. Platen. Cosmic Voids: Structure, Dynamics and Galaxies. International Journal of Modern Physics Conference Series, 1:41-66, 2011. .

R. van de Weygaert \& W. Schaap. The Cosmic Web: Geometric Analysis. In V. J. Martínez, E. Saar, E. Martínez-González, and M.-J. Pons-Bordería, editors, Data Analysis in Cosmology, volume 665 of Lecture Notes in Physics, Berlin Springer Verlag, pages 291-413, 2009. .

R. van de Weygaert \& E. van Kampen. Voids in Gravitational Instability Scenarios - Part One Global Density and Velocity Fields in an Einstein - De-Sitter Universe. MNRAS, 263:481, July 1993.

I. B. Zeldovich, J. Einasto, \& S. F. Shandarin. Giant voids in the universe. Nature, 300:407-413, December 1982. . 